\title{
Improved attenuated total reflectance technique for the investigation of dielectric surfaces
}

\author{
$S$. Herminghaus and $P$. Leiderer \\ Institute für Physik, Johannes Gutenberg Universität Mainz, Federal Republic of Germany
}

(Received 10 August 1988; accepted for publication 28 October 1988)

\begin{abstract}
Guided wave surface polaritons (GWSPs) propagating in a dielectric slab adjacent to a metal surface are investigated both theoretically and experimentally. It is shown that GWSPs can be used for high-resolution analysis of dielectric surfaces by means of the well-known attenuated total reflectance technique. From our experimental results we conclude that changes in the system equivalent to the adsorption of $1 / 30$ of a monolayer can easily be resolved. A wellestablished surface characterization method, which had been restricted to few systems up to now, is thus immensely generalized.
\end{abstract}

Optically excited surface plasmons (SPs) have been used by many authors for metal surface characterization by means of the attenuated total reflectance (ATR) technique. ${ }^{1-3}$ Under certain conditions, the SPs at the metal surface under investigation can be excited resonantly, which gives rise to a sharp dip of the reflected light intensity. The shape of the resonance curve carries information about the structure of the surface and its coverage with adsorbed films. For instance, a dielectric coverage results in a shift of the resonance angle $\theta_{0}$, which is roughly given by

$$
\Delta \theta_{0}=b(\epsilon-1) D,
$$

where $D$ and $\epsilon$ are the thickness and dielectric constant of the covering layer, respectively, and $b$ is of the order of 0.1 $\mathrm{deg} / \mathrm{nm} .{ }^{4}$ On a silver surface, the $\mathrm{SP}$ resonance is exceptionally narrow, so the adsorption or desorption of a layer with thickness of the order of $1 \AA$ is well detectable. ${ }^{4.5}$ Besides its high sensitivity, this method has considerable advantages compared to other optical surface analysis techniques, e.g., its stability against strain-induced birefringence in the optical apparatus and the simplicity of the setup. However, it lacks versatility, because the $\mathrm{SP}$ resonances are not equally sharp in all metals; in fact, by far, most of the work done in the field of SPs deals with noble metal surfaces for this reason. Consequently the use of SPs for surface analysis has been restricted to few systems up to now, in fact, mainly to the silver surface.

In this letter we propose an improved ATR technique, which involves so-called guided wave surface polaritons (GWSPs), and can be used to characterize the surfaces of a wide class of dielectric materials. In our type of setup, the dielectric, whose surface is to be investigated, forms a film on a metal surface, thick enough for consideration as a bulk sample ( $>0.5 \mu \mathrm{m}$ ). In the film, guided waves can propagate which couple to the free-electron plasma in the metal, forming the GWSPs. ${ }^{6}$ These can be excited optically in the same way as SPs using TM polarized light (see inset in Fig. 1), yielding similar dips in the reflected light intensity when the resonance condition is satisfied. It is the aim of this letter to show that the sensitivity of these GWSP modes to changes of the dielectric surface, e.g., the adsorption of an additional dielectric layer, is comparable to that of the SPs at the metal surface.
We have investigated numerically an ATR configuration, consisting of a glass prism ( $\epsilon=2.2955)$, a $53 \mathrm{~nm}$ silver layer $[\epsilon=-18.35+0.55 i$ (Ref. 7) $]$, a dielectric overlayer $[\epsilon=2.179$ corresponding to bulk polymetylmethacrylate (PMMA)], and an adjacent vacuum half-space. The adsorption of dielectric material onto the PMMA overlayer was modeled by slightly changing the overlayer thickness. Calculations were carried out using the transfer matrix method.$^{8-10}$ A divergence of the detecting TM laser beam $(\lambda=632.8 \mathrm{~nm})$ of $0.06^{\circ}(\approx 1 \mathrm{mrad})$ has been taken into account by convolution of the calculated reflectivity curve $R(\theta)$ with a Gaussian profile. In Fig. 1 the calculated reflectivity of the layer system is plotted as a function of the angle of incidence for a thickness of the dielectric layer of $D=1.2$ $\mu \mathrm{m}$. It shows four GWSPs; the SP resonance can no longer be excited at that thickness (cf. Fig. 2).

The dependence of the number and the position $\theta_{0}$ of the GWSP resonances on the thickness of the waveguide layer is illustrated in Fig. 2. Obviously, for every GWSP mode, its resonance position is most sensitive to changes of the thickness of the layer when $\theta_{0}$ is small, i.e., in the vicinity of the total reflectance angle. We now define the sensitivity $S$ of a

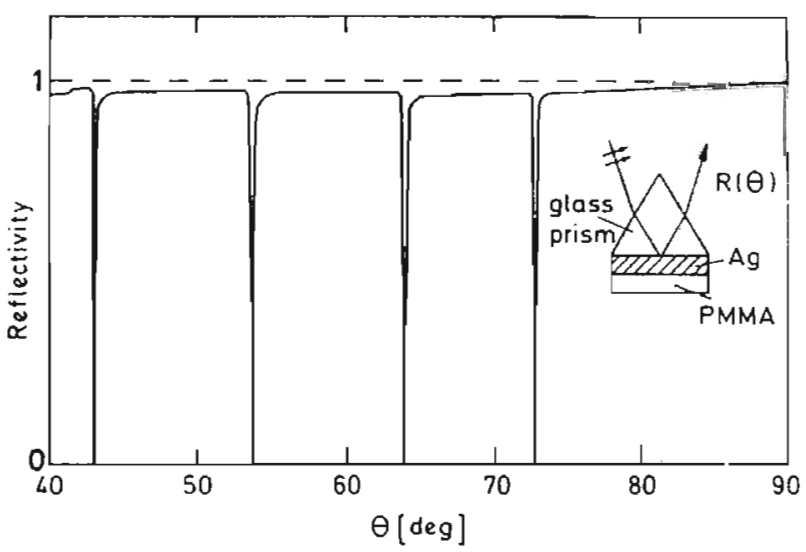

FIG. 1. Calculated reflectivity (for TM polarized light) vs the angle of incidence for the ATR configuration shown in the inset. The four resonances are the GWSPs with mode indices $4,3,2$, and 1 , from left to right. The thickness of the PMMA layer is $1.2 \mu \mathrm{m}$. 


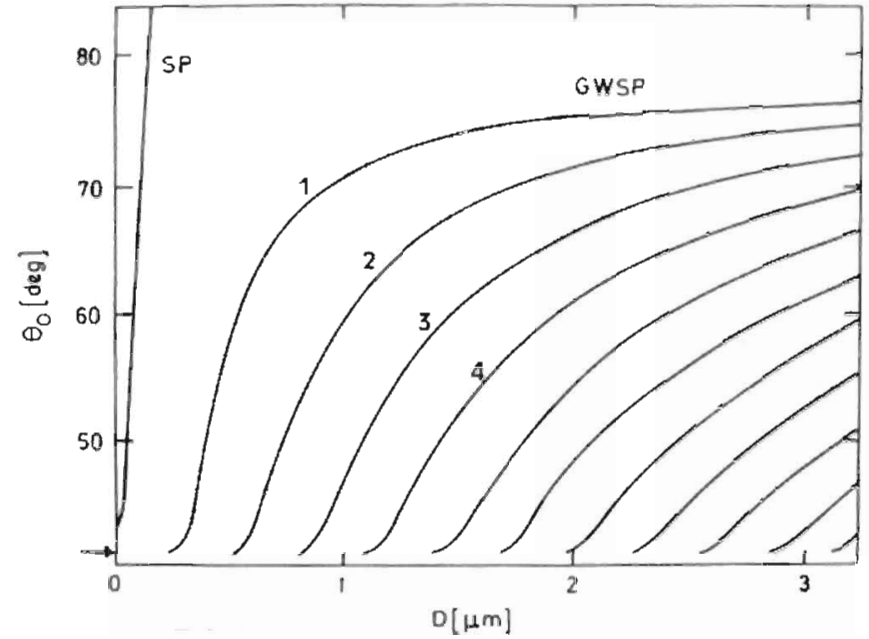

FIG. 2. Dependence of the resonance angles $\theta_{0}$ of the SPs and the GWSPs on the thickness of the waveguide layer (PMMA). The angle of total reflectance is marked by an arrow. The numbers denote the mode indices for the first four GWSPs.

GWSP resonance by the product of the resonance angle shift and the second derivative of the reflectivity curve taken at the resonance angle $\theta_{0}$ (this is justified by the experimental procedure described below ):

$$
S=\left.\frac{d \theta_{0}}{d D} \frac{d^{2} R}{d \theta^{2}}\right|_{\theta_{n}}=\frac{d^{2} R}{d \theta d D} .
$$

This quantity describes the capability of a GWSP to resolve a change in the waveguide thickness $D$ and, consequently, a thin additional dielectric coverage.

A survey of the calculated sensitivity of a large number of modes is given in Fig. 3. The angle of incidence was fixed to $43^{\circ}$ in this case. On increasing the waveguide thickness, the GWSP resonances appear successively; their mode indices are given on top of the diagram. The vertical scale shows the quantity $S$. The two sets of data points show the effect of the laser beam divergence on $S$. This reflects the

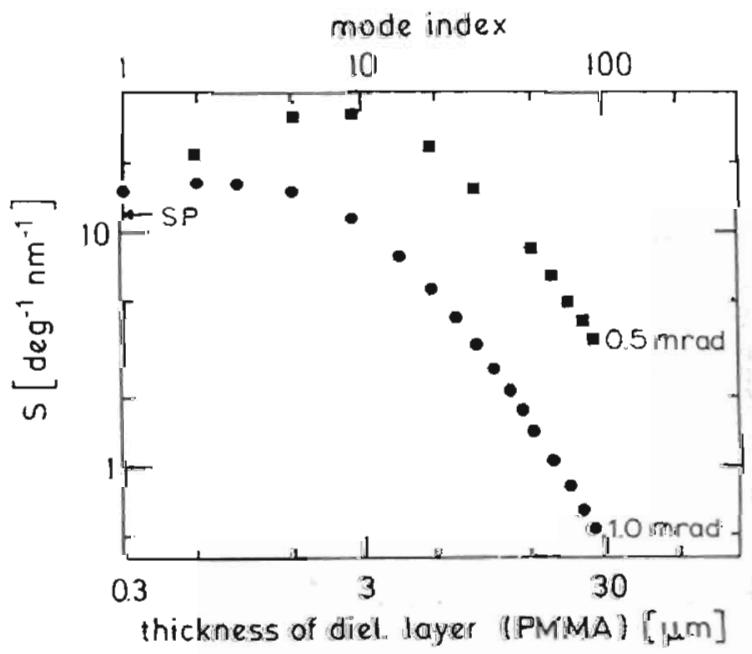

FIG. 3: Log-log plot of the calculated sensitivity $S$ of the GWSP nearest to the total reflectance angle (at $43^{\circ}$ ) ys the waveguide thickness. The corresponding mode index can be taken from the top scale. Note that not all modes are shown.
TABLE 1. Dielectric coverage equivalent to replacement of surrounding gas.

\begin{tabular}{ccccc}
\hline \hline $\begin{array}{c}\text { Coating material } \\
\text { (waveguide) }\end{array}$ & $\epsilon$ & $\begin{array}{c}d \\
(\mu \mathrm{m})\end{array}$ & Mode index & $\begin{array}{c}\text { Equivalent } \\
\text { coverage }(\AA \AA) \\
\text { for } \epsilon=2\end{array}$ \\
\hline$\ldots$ & $\ldots$ & 0 & SP & 2.43 \\
PMMA & $2.179^{\circ}$ & 0.6 & 2nd GWSP & 2.04 \\
mica & $2.34^{\mathrm{b}}$ & 17.4 & 65th GWSP & 2.0 \\
glass & 2.296 & 41.4 & 149th GWSP & 2.1 \\
\hline
\end{tabular}

"Taken from bulk PMMA.

${ }^{b}$ A veraged: $n_{x}=1.527 ; n_{z}=1.556$ (Ref. 11).

extrcme sharpness of the higher order GWSP modes: the 5th and the 80th mode, for example, have a full width at half maximum of $0.06^{\circ}$ and $0.0025^{\circ}$, respectively (when the resonance angle is in the vicinity of $43^{\circ}$ ). The shift $d \theta_{0} / d D$ tends to zero with increasing mode index, yielding a decrease of $S$. For low mode indices, however, the sensitivity $S$ is comparable to, or even exceeds, that of the surface plasma resonance.

For an experimental test of the sensitivity of GWSPs on coverage of the dielectric surface, one would need an independent and reliable method to vary and control the coverage of suitable adsorbates on a monolayer scale. Without such a technique presently at hand, we have chosen a simple alternative procedure: The air adjacent to the surface under investigation was replaced by helium gas, which changed the dielectric constant of the whole half-space from $\epsilon=1.000276$ to $\epsilon=1.000035$. " As can be shown numerically, this has just the opposite effect on the resonances as covering the waveguide layer with approximately $2 \AA$ Af a dielectric material with $\epsilon=2$ (the exact value of the equivalent coverage depends on the mode number and the dielectric constant of the waveguide layer; it can be taken from Table I). The experimental setup was a standard ATR configuration as described earlier (see for instance Ref. 12). A He-Ne laser $(632.8 \mathrm{~nm})$ was used for the GWSP excitation. We detected the shift of the resonances by a lock-in technique: the angle of incidence was modulated periodically and the reflected intensity was fed into a lock-in amplifier, yielding an output voltage proportional to the slope $d R / d \theta$ of the reflectivity curve. A shift $\Delta \theta_{0}$ of the resonance angle hence resulted in a signal proportional to $\Delta \theta_{0}$ and to the quantity $S$ defined in the previous section. Experiments were carried out with the three different dielectric coatings listed in Table I. The PMMA film had been obtained by deposition from a solution of PMMA in acetone. The two other samples were prepared by evaporating the silver film onto the free-standing dielectric slabs; the silver-coated side of the slab was then attached to the coupling glass prism with immersion fiuid.

Experimental results are shown in Fig. 4 for the GWSPs on the PMMA, mica, and glass waveguides and, for comparison, for the SPs on bare silver. The lock-in signal is plotted versus time. Obviously a GWSP resonance angle shift corresponding to the adsorption of about one monolayer onto a bulk dielectric surface can be detected with a sensitivity comparable to that of the SP resonance. For example, in 


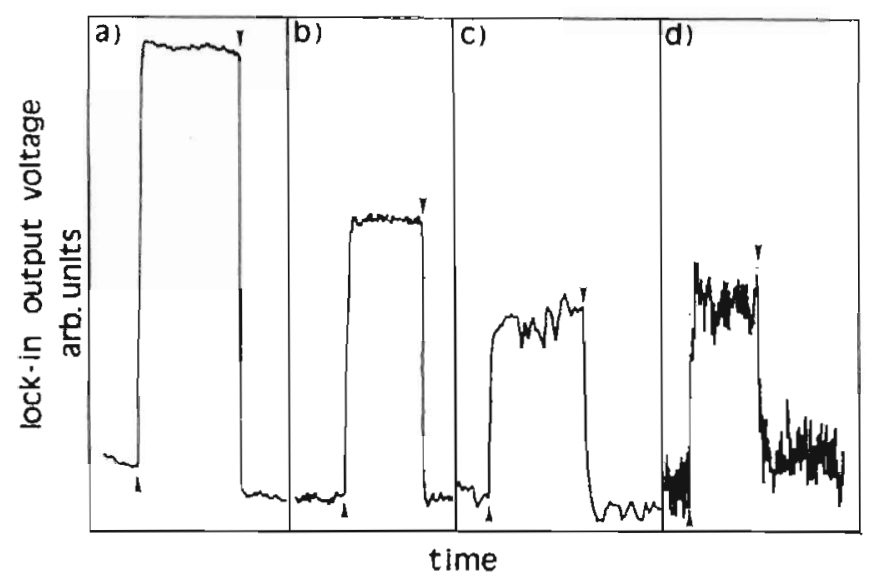

FIG. 4. Lock-in signals obtained on replacing the air in the vicinity of the illuminated sample region by helium: (a) SP on the bare silver surface; (b) 2nd GWSP in $0.6 \mu \mathrm{m}$ of PMMA; (c) $65 \mathrm{th}$ GWSP in $17.4 \mu \mathrm{m}$ of mica; (d) 149 th GWSP in $41.4 \mu \mathrm{m}$ of glass. The iock-in time constant was $1 \mathrm{~s}$. The vertical scale is different for the four signals in order to show them all together in one diagram. The arrows denote the replacement of air by helium (1) and vice versa (!). The time scale is about $1 \mathrm{~min}$ for each trace.

Fig. 4(b) the "noise" corresponds to about $1 / 30$ of a monolayer. Furthermore, there is still appreciable sensitivity when the waveguide is formed by a $0.04-\mathrm{mm}$-thick glass plate [Fig. 4(d)]. The general tendency of a decrease of sensitivity with increasing mode index, as suggested by the above theory, is confirmed by the experiment.
In conclusion, GWSPs can be used for extremely sensitive characterization of the surfaces of a wide class of bulk dielectric materials. Any dielectric which can be prepared as a film thicker than about half a micron (necessary for carrying guided waves) adjacent to a silver fil $\mathrm{m}$ can be investigated. Our experiment suggests that the adsorption of small fractions of a monolayer onto the dielectric film can be detected, and further improvement appears to be possible (at least by reducing the divergence of the detecting laser beam). The resulting improved ATR technique proposed in this letter thus opens a wide field of application to a method which had been restricted to very few systems up to now.

This work has been supported by the Deutsche Forschungsgemeinschaft (Sonderforschungsbereich 252).

'E. Kretschmann, Opt. Commun. 10, 353 (1974).

${ }^{2}$ A. Humbert and J. Hanus, Surf. Sci. 129, 265 (1983).

'E. Fontana and R. H. Pantell, Phys. Rev. B 37, 3164 (1988).

II. Pockrand, Surf. Sci. 72, 577 (1978).

'S. Puderbach, S. Herminghaus, and P. Leiderer, Phys. Lett. A 130, 401 (1988).

'M. S. Tomas and Z. Lenac, Opt. Commun. 55, 267 (1985).

'P. B. Johnson and R. W. Christy, Phys. Rev. B 6, 4370 (1972).

${ }^{*}$ P. H. Bernick, in Physics of Thin Films, edited by G. Haas (Academic, New York, 1963), Vol. 1, p. 69.

"M. Born and E. Wolf, Principles of Optics (Pergamon, Oxford, '975).

"'Z. Knittl, Optics of Thin films (Wiley, New York, 1976).

''J. Bartels, H. Borchers, H. Hausen, K. Hellwege, K. Schäfer, and E. Schmilt, Landoldi-Börnstein, 6thed. (Springer, Berlin, 1982), Vol. 11/8, pp. $2 / 141,6 / 871$, and $6 / 878$.

'2E. Kretschmann and H. Raether, Z. Naturforsch. Teil A 23, 2135 (1968). 